\title{
On Bubble Sizing in Water by Ultrasound
}

\author{
https://doi.org/10.3991/ijim.v11i2.6590 \\ W. B. Hussein \\ The British University in Egypt, Cairo, Egypt \\ walid.hussein@bue.edu.eg \\ S. A. Essmat \\ The British University in Egypt, Cairo, Egypt \\ sarah.akramabue.edu.eg \\ Nestor B. Yoma \\ Universidad de Chile, Santiago, Chile
}

\begin{abstract}
Classifying bubbles in liquids is a crucial problem that is demanded within multiple fields. This paper discusses a new method for classifying bubble sizes in non-contact and inexpensive approach using ultrasound analysis. Exploiting the principle of buoyancy, free rising bubbles with larger volumes elevate faster to the surface compared to the smaller ones, given that they have the same densities. An envelope detector is proposed which tracks the changes in the ultrasound signals reflected by the bubbles when they cross the ultrasound field. These changes in the reflected signals are distinctive for the sizes under consideration. Relevant spectral and linear predictive coding features that represent the distinct characteristics are extracted. These features are fed to a feed-forward artificial neural network to successfully classify air bubbles according to their sizes with an accuracy of $98.8 \%$. This method provides promising applications to be implemented in industrial, biomedical and environmental fields.
\end{abstract}

Keywords-Artificial Neural Networks, Bubble Sizing, Feature Extraction, Ultrasound Analysis.

\section{Introduction}

Gas bubbles sizing finds application in multiple fields including industrial, environmental, and biomedical applications [1]. Methods for characterization of gas bubbles typically exploit high-speed photography, passive acoustic detection, capillary suction probes, optical waveguide sensors, and endoscopic optical probes [2].

Lin et al. [3] used three parallel planes of laser light between two photo detectors and proposed a pulse displacement method to make measurements of refractive index, size and velocity of bubbles. The detectors sense multiple refracted (and reflected) beams as the bubbles travel. Barigou and Greaves [4] conducted experiments by capillary suction and placement of photo detectors. Kawaguchi et al. [5] presented an 
interferometer laser imaging technique to size bubbles and droplets. They estimated diameters as low as $200 \mu \mathrm{m}$.

Vazquez et al. [2] measured volume and radius of gas bubbles in quiescent water using three methods i.e. photographic and acoustic schemes, together with the laboratory-standard inverted funnel or the capillary suction probe method. The authors reported that the standard technique gave a $0.5 \%$ repeatability error with a 50 -bubble set, while the photographic method provided accuracy between $86 \%$ and $99 \%$ with a $5 \%$ repeatability error. The passive acoustic approach gave an accuracy of $97 \%$ and $99 \%$ with a repeatability of $0.3 \%$. They concluded that the optical and acoustic methods both provided accurate estimations and suggested the use of a piezo-electric element instead of a hydrophone. The optical method was limited by the lighting conditions and required the purity of the fluid medium.

Majority of the acoustic techniques for bubble characterization rely on the ability of the bubble to oscillate with a resonance frequency that is approximately inversely proportional to its radius [1]. However, the use of a single frequency has been shown to have limitations, for example, the masking of small bubbles in the presence of large ones. Therefore, researchers often utilize two frequencies to exploit the nonlinearity related to the high amplitude pulsations [1] [6]. Wu and Chahine [7] developed the "Acoustic Bubble Spectrometer" to estimate bubble size distribution by measuring frequency-dependent attenuations and phase velocities of the sound waves. Results obtained with this method were consistent with those from high speed video photography. Most studies generally consider a simplistic model for resonance frequency and ignore the effects related to the elastic properties of bubble walls, stiffness, inertia, and the proximity of the boundaries, multi-bubble effects [8].

In this paper a novel pattern classification technique for bubble sizing is presented. The approach exploits two-dimensional (2D) patterns representing the trace of bubbles when they cross an ultrasound beam. The $2 \mathrm{D}$ patterns relate to the average spectrum of the reflected ultrasound signal from a single bubble or a train of bubbles of the same size.

Frequency-domain based features i.e. fast Fourier transform (FFT) and linear predictive coding (LPC) are fed to an artificial neural network (ANN) which is trained to classify different bubble sizes. The proposed scheme benefits from the advantage that it is able to process multiple bubbles simultaneously, masking interference among bubbles are potentially minimized compared to the resonance frequency based methods, and the transmitter-receiver electronics is highly simplified because only one sinusoidal component is used.

\section{Experimental Setup}

The setup, as displayed in Fig. 1 . is a $22 \times 24 \times 35 \mathrm{~cm}$ acrylic with a wall thickness of $1 \mathrm{~cm}$. The tank was filled by water at $25 \pm 0.5$ Celsius, with a surface tension of $72.9 \times 10^{-3} \mathrm{~N} / \mathrm{m}$, density of $998 \mathrm{Kg} / \mathrm{m}^{3}$, and viscosity of $0.89 \times 10^{-3} \mathrm{~kg} /(\mathrm{m} / \mathrm{s})$. Air bubbles were supplied at the bottom of the tank through a capillary tube connected to a pressure pump, Omega FMA5400/5500, with a controllable mass flow rate. The capil- 
lary tube delivers the bubbles to the tank with their terminal speed to ensure that bubbles velocity while elevating in the tank is function only in the bubbles dimensions. Elevated bubbles cross the ultrasound field created by the emitter transducer, and reflect signals to the orthogonally placed receiver transducer.

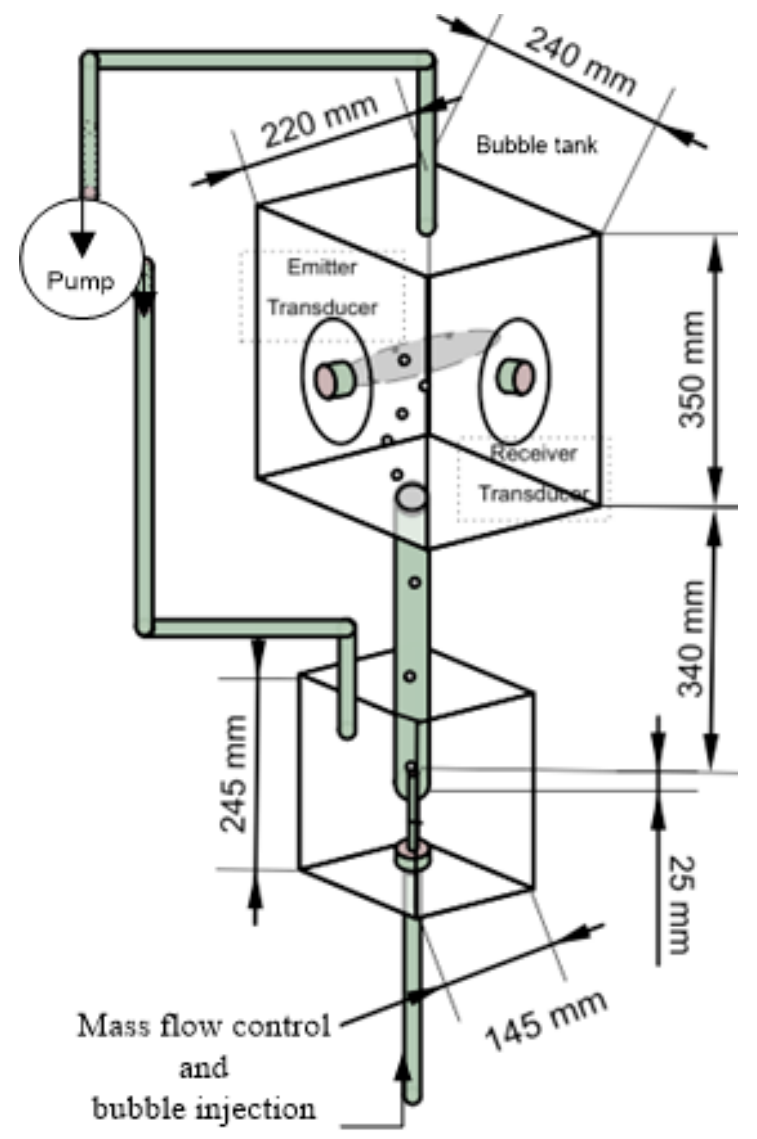

Fig. 1. Schematic diagram for the experimental setup employed to collect the signals reflected by the free-rising air bubbles in water.

The experiments were separately ran at three different air mass flow rates $(10,30$, and 60$) \mathrm{mL} / \mathrm{min}$., producing three different bubble sizes of average diameters of $(2.5$, 5 , and 6.5$) \mathrm{mm}$; respectively. In this paper, it will be referred to these sizes by $\mathrm{S}_{1}$ for the small bubbles, $\mathrm{S}_{2}$ for the medium bubbles, and $\mathrm{S}_{3}$ for the large bubbles.

\section{Envelope Detection}

The ultrasound signals were generated at frequency of $1 \mathrm{MHz}$. this frequency was selected to attain a signal with a wavelength able to detect as low as the smallest bub- 
ble $S_{1}$. These signals were went through an amplifier to raise their powers before fed into the emitter transducer. A full wave rectifier (i.e., impedance-matching transformer) was connected to match the impedance between the amplifier and the transducer. Signals are afterwards passed through a band pass filter with a band [875 - 1125] kHz to filter out all attached noises and then re-amplified. An analog-to-digital converter is utilized so that the data can be processed by a computer. The envelope detection procedure is shown in Fig. 2.

The envelope detection process aims to trace the outer contours of the signals reflected by the bubbles while crossing the ultrasound field during their elevation. These contours are believed to have distinctive characteristics due to the size of the elevated bubbles. Larger bubbles would rise faster to the surface, modifying the signal's envelope in a different way in comparison to the signal's envelope for the smaller bubbles.

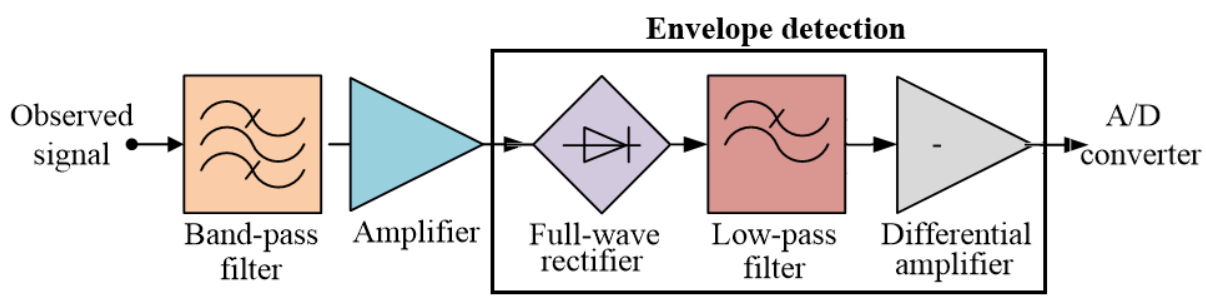

Fig. 2. Block diagram shows the envelope detection procedure for the observed signal.

\section{$4 \quad$ Signal Processing}

The Reflected signals were sampled at $180 \mathrm{kHz}$ and collected at the receiver transducer. The analysis of these signals over all the experiments and bubble sizes - and with the aid of video recordings - indicated that the frequency band which contains significant information (i.e., bubbles activity) is the one lie between [0 - 55] $\mathrm{kHz}$. Therefore, the signals are low-pass filtered with a cut-off frequency of $55 \mathrm{kHz}$ to remove any attached noise.

As mentioned before, the envelope of the reflected signal represents the size of the source bubbles. However, the duration of these signals are determined according to how many bubbles are elevating during the experiment and cross the ultrasound field, as shown by example in Fig.3. Therefore, the analysis in time domain may be misleading.

Alternatively, the frequency domain representation of the reflected signal is not sensitive to its duration, and it provides the frequency contents of the corresponding signal regardless its duration. Since the signal in time domain is different for each bubble size, its frequency domain representation behaves in the same manner, and can be used to distinguish between experiments of different bubble sizes, as given in Fig.4. 


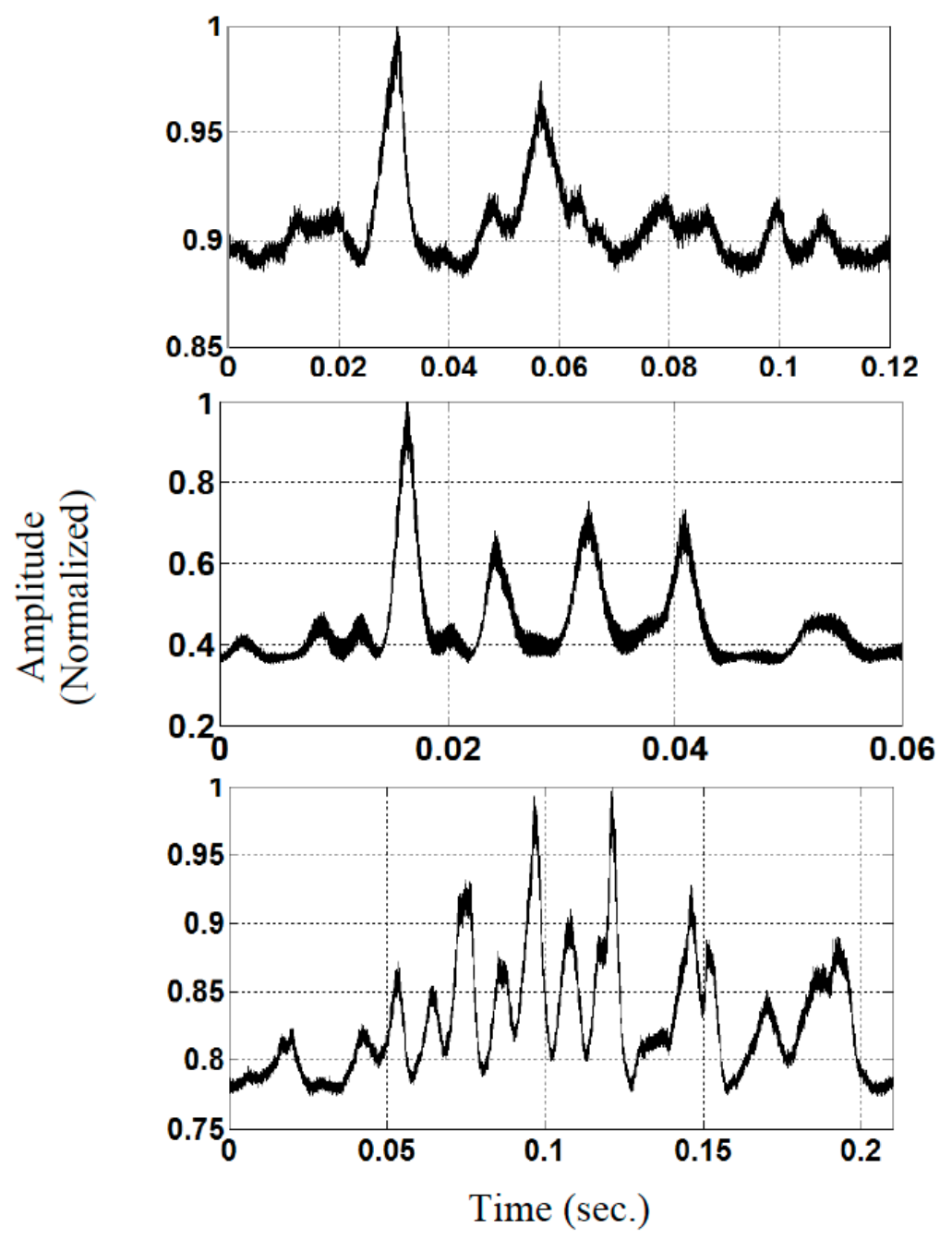

Fig. 3. An example for the time domain representation of the signals of different durations recorded at the receiver. From top to bottom, signals for bubbles of sizes $\mathrm{S}_{1}, \mathrm{~S}_{2}$, and $\mathrm{S}_{3}$; respectively.

From different approaches, fast Fourier transform (FFT) was employed in this paper to transform the signals into their frequency domain representation [9]. The signal was divided into short overlapped segments with a length equals $10 \%$ of the signal's length and an overlapping percentage of 50\%. Hamming window function is used to reduce the spectral leakage in the resultant spectrums. The bigger bubbles $\left(\mathrm{S}_{3}\right)$ move faster to the surface of water and thus its reflected signal has the maximum peak frequency of $49 \mathrm{kHz}$. It decreases to $44.5 \mathrm{kHz}$ for $\mathrm{S}_{2}$ and $42 \mathrm{kHz}$ for $\mathrm{S}_{1}$. This validates the hypothesis that the faster the bubble rises to the surface due to its size, the perturbation in bubble trajectory and shape will contain higher frequency components. 


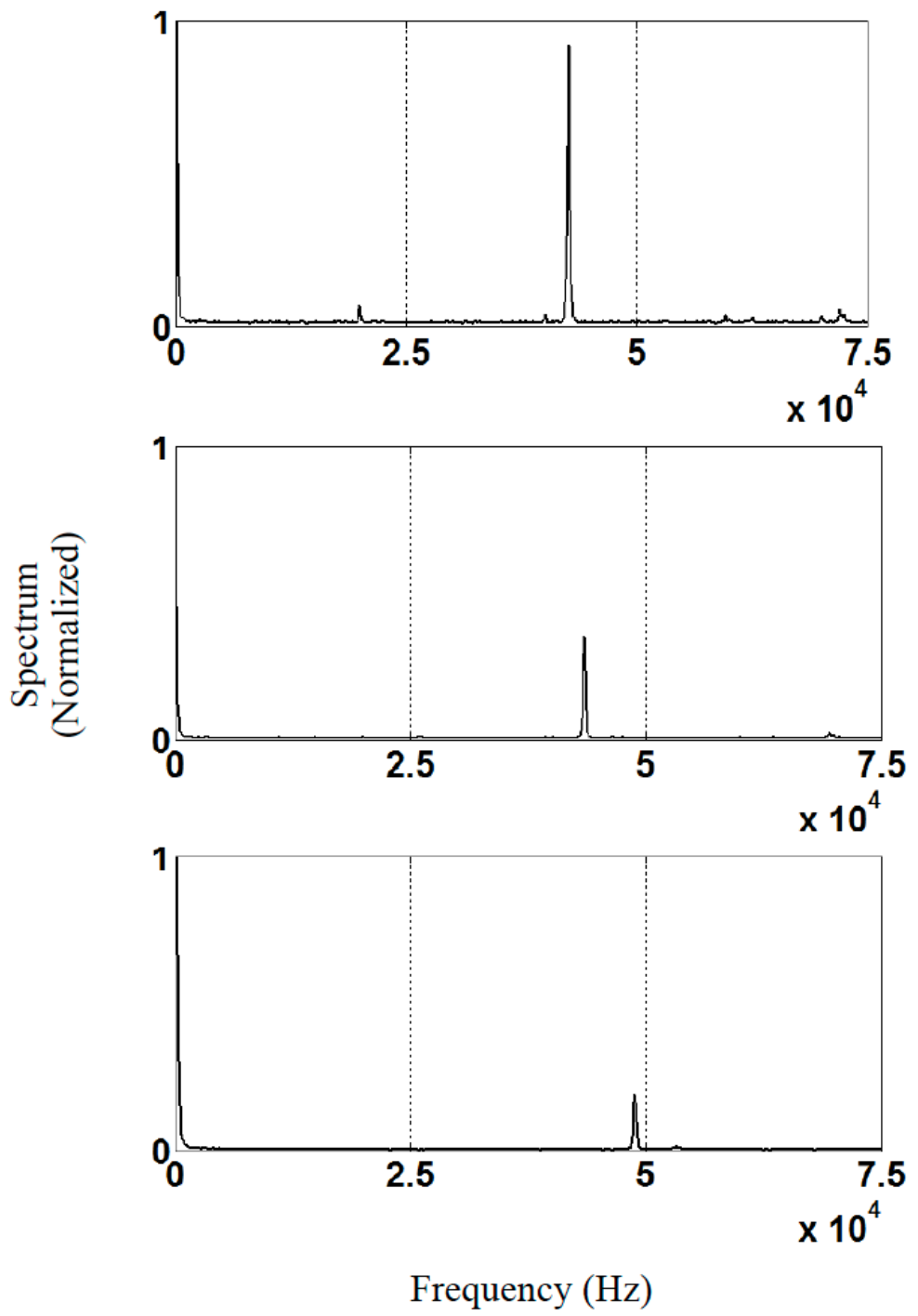

Fig. 4. The frequency domain representation for the signals presented in Fig. 3, using Fast Fourier Transform (FFT) with a Hamming window and 50\% overlapping.

\section{$5 \quad$ Feature Extraction}

The Frequency domain based features i.e. spectral centroid, entropy, and crest factor are extracted from the spectrum of the signals [10]. Spectral centroid represents the frequency where half of the energy in the pattern is achieved, while entropy estimates the total loss of the pattern energy. Crest factor measures the compactness of the spectrum. The features are mathematically written as: 


$$
\begin{gathered}
\text { Spectral Centroid }=\frac{\sum_{m=1}^{\mathrm{M}} n \cdot|X(m)|}{\sum_{m=1}^{\mathrm{M}}|X(m)|} \\
\text { Spectral Entropy }=-\sum_{m=1}^{\mathrm{M}} p m f(m) * \ln (p m f(m)) \\
\text { Spectral crest factor }=\frac{\max (|X(m)|)}{\frac{1}{\mathrm{M}} \sum_{m=1}^{\mathrm{M}}|X(m)|}
\end{gathered}
$$

Where $p m f$ is the probability mass function $\left[p m f(m)=\frac{|X(m)|}{\sum_{m=1}^{\mathrm{M}}|X(m)|}\right]$.

LPC on the other hand gives an insight into the spectral envelope of the signal by estimating the LPC coefficients [11]. In this paper, LPC analysis with 10 coefficients was performed. The LPC coefficients were used alongside the other spectral features.

\section{Results and Discussion}

The For each bubble size, 112 experiments were ran resulted in $(112 \times 3=336)$ signals. Afterwards, the 3 FFT features and the 10 LPC coefficients were extracted to represent the spectrum's characteristics of each signal.

For the 3 FFT features, spectral centroid, crest factor, and entropy, it was noticed that they are changing with the change of bubble size. The key statistics, mean and standard deviation, were calculated for the FFT features over the 112 experiments of each bubble size, and presented in Fig.5. While both the spectral centroid and crest factor increase with the increment of the bubble size, it is the opposite for spectral entropy. This is due to the physical meaning of entropy, which represents loss of energy, and larger bubbles reflect signals with higher energy than those reflected by smaller bubbles.

Meanwhile, the spectral envelope obtained by LPC coefficients was also different for each size, as displayed in Fig.6. This spectral envelope roughly indicates how the peak frequency is high for signals reflected by larger bubbles, and reduces as bubbles become smaller.

A feed-forward multilayer perceptron ANN was designed using the back propagation algorithm to develop a bubble classifier. The input layer was made of 13 neurons for the 3 FFT features and the 10 LPC coefficients. One hidden layer with five neurons was selected while the output layer contains three neurons for the classification's classes $\left\{A_{1}, A_{2}, A_{3}\right\}$, with values $\{1,0,0\}$ for $S_{1},\{0,1,0\}$ for $S_{2}$, and $\{0,0,1\}$ for $S_{3}$. The transfer functions for the neurons should ensure the smallest total mean square error (MSE) while training of the network. Iterations on type of the activation functions had been applied using LOGSIG and TANSIG activation functions, until the best results are obtained [12]. 
Paper-On Bubble Sizing in Water by Ultrasound
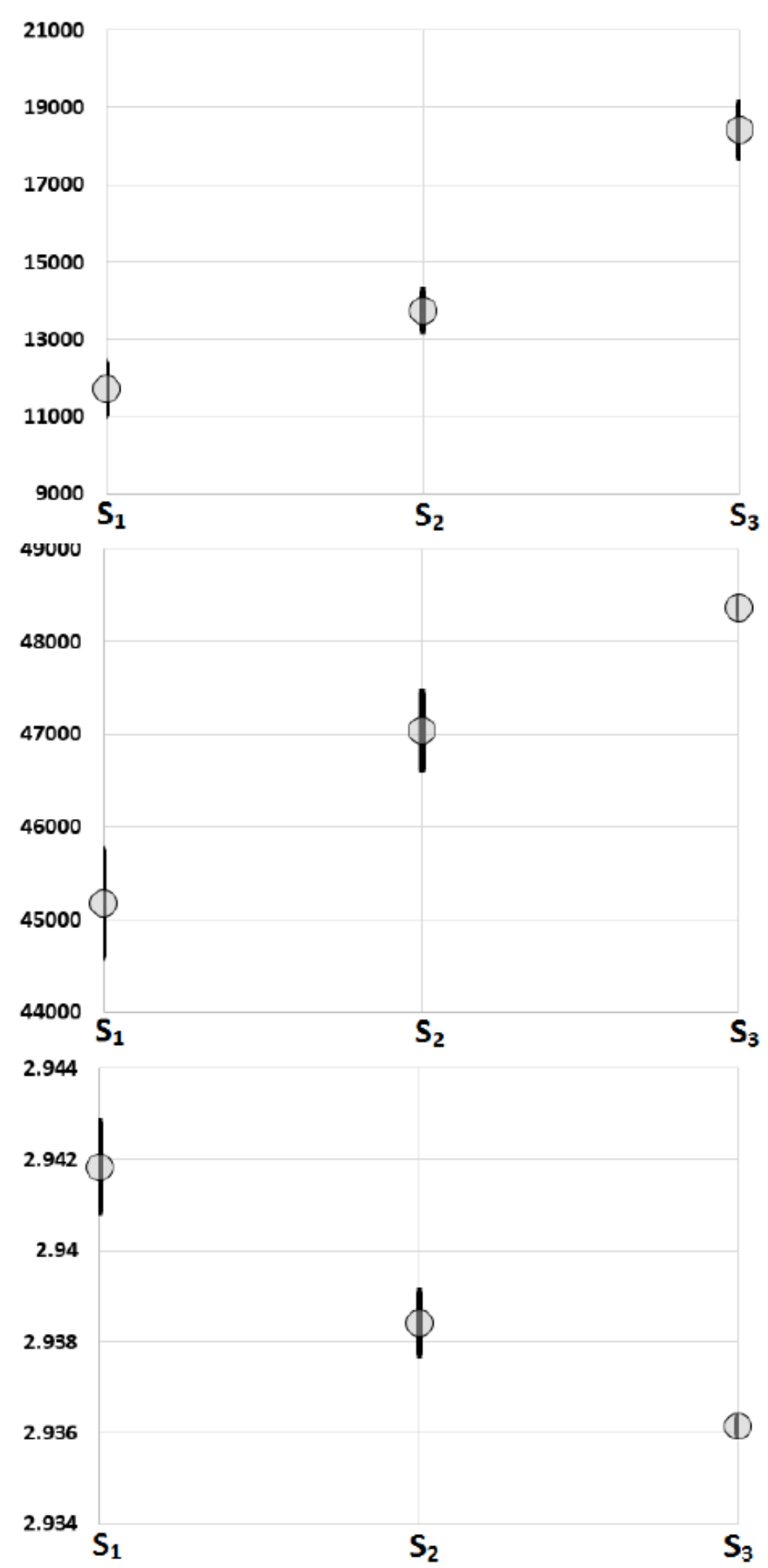

Fig. 5. Mean and standard deviation of the extracted FFT features for signals of the three sizes. (top) spectral centroid, (middle) spectral crest factor, and (bottom) spectral entropy. 


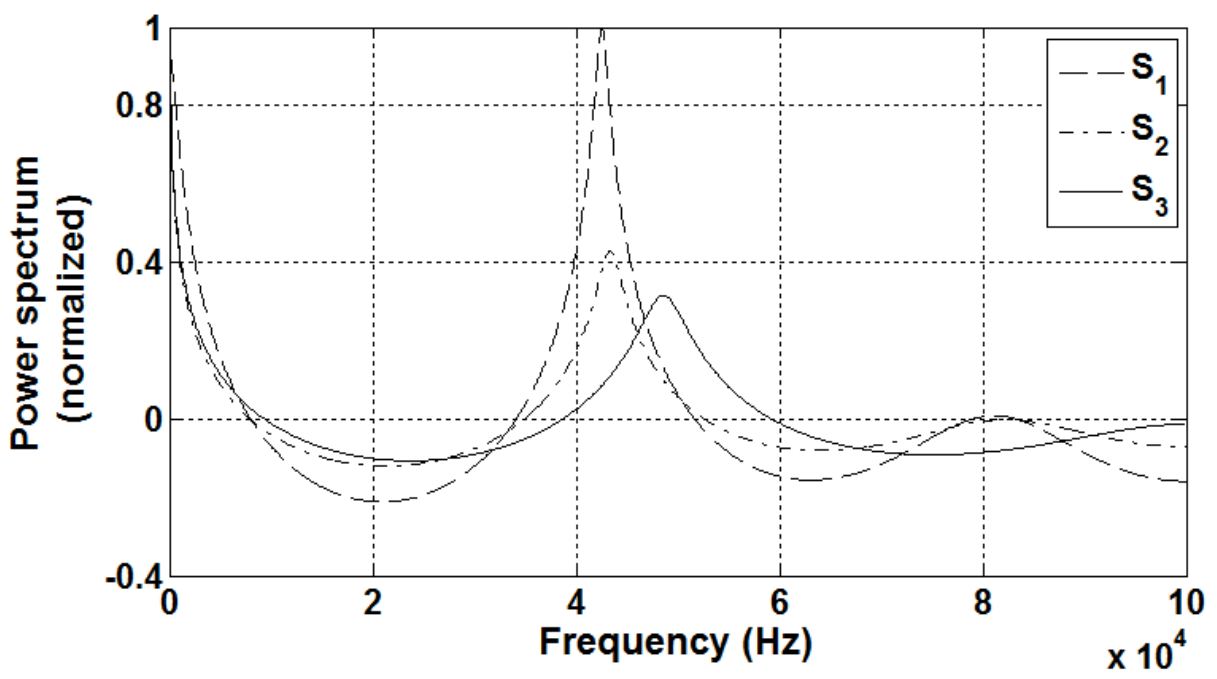

Fig. 6. Spectral envelope obtained by 10 coefficients LPC analysis.

Before training, the network inputs were scaled to their mean-centered form so that the network sets the same priority to each input. The 13 parameters at the input layer were obtained for the 336 signals, resulting in a database matrix of size 336 samples $\times$ 13 parameters. This database was divided into three sets. The first set contains $50 \%$ of the total data and is used to train the network, the second set contains $25 \%$ and is used to validate the resultant network and to measure its generalization. While the third set contains the remaining $25 \%$ and is used to test the selected network.

After 50 iterations, MSE reached its minimum value of 0.071 . This network was then utilized to classify the testing data into their associated classes, as shown in table (1), giving a classification accuracy $\mathrm{ACC}=98.8 \%$. The developed network correctly classified the signals of $\mathrm{S}_{2}$ and $\mathrm{S}_{3}$ into their target classes, while wrongly classified one signal of $\mathrm{S}_{1}$ to the class of $\mathrm{S}_{2}$.

Table 1. Accuracy of the developed network to classify the testing signals.

\begin{tabular}{|c|c|c|c|c|}
\hline & \multicolumn{3}{|c|}{ Target class } \\
\hline & & $S_{1}$ & $\mathbf{S}_{2}$ & $\mathbf{S}_{3}$ \\
\hline \multirow{3}{*}{ 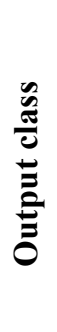 } & $\mathbf{S}_{1}$ & $\begin{array}{c}28 \\
(100 \%)\end{array}$ & $\begin{array}{c}1 \\
(3.6 \%)\end{array}$ & $\begin{array}{c}0 \\
(0.0 \%)\end{array}$ \\
\hline & $\mathbf{S}_{\mathbf{2}}$ & $\begin{array}{c}0 \\
(0.0 \%)\end{array}$ & $\begin{array}{c}27 \\
(96.4 \%)\end{array}$ & $\begin{array}{c}0 \\
(0.0 \%)\end{array}$ \\
\hline & $\mathbf{S}_{\mathbf{3}}$ & $\begin{array}{c}0 \\
(0.0 \%)\end{array}$ & $\begin{array}{c}0 \\
(0.0 \%)\end{array}$ & $\begin{array}{c}28 \\
(100 \%)\end{array}$ \\
\hline
\end{tabular}


The number of classes can be increased when more than 3 bubble sizes are under consideration, provided that the minimum bubble size is comparable to the ultrasound wavelength. Observe that each experiment involves one or group of bubbles of the same sizes.

It is worth highlighting that the proposed method would start having difficulties if there are objects preventing the receiver from capturing the reflected signals. In real applications, the above mentioned scenario seems unlikely.

\section{Conclusion}

A novel pattern classification approach for bubble sizing was proposed. The technique exploited 2D patterns, generated by the reflected ultrasound signal that represent the trace of bubbles when they cross the measurement area. Frequency-domain based features were extracted and an artificial neural network was designed and trained to classify different bubble sizes. Results suggest that the proposed method can lead to a classification accuracy as high as $99 \%$.

\section{Acknowledgment}

This research was partially funded by the Chilean national association for investigation in science and technology (conicyt), project Anillo Act1120, Chile.

\section{References}

[1] T. G. Leighton, “The acoustic bubble”, Academic Press, London, UK, 1994, pp. 129-152. https://doi.org/10.1016/B978-0-12-441920-9.50008-0

[2] A. Vazquez, R.M. Sanchez, E. Salinas-Rodriguez, A. Soria, R. Manasseh, "A look at three measurement techniques for bubble size determination", Experimental Thermal and Fluid Science, volume 30,2005, pages 49-57. https://doi.org/10.1016/j.exptherm flusci.2005.03.018

[3] S. M. Lin, D. R. Waterman and A. H. Lettington, "Measurement of droplet velocity, size and refractive index using the pulse displacement technique", Measurement Science \& Technology, volume 11, 2000.

[4] M. Barigou and M. Greaves, "A capillary suction probe for bubble size measurement", Measurement Science \& Technology, volume 2, pages 318-26, 1991. https://doi.org/10.1088/0957-0233/2/4/009

[5] T. Kawaguchi, Y. Akasaka and M. Maeda, "Size measurements of droplets and bubbles by advanced interferometric laser imaging technique", Measurement Science \& Technology, volume 13, pages 308-16, 2002. https://doi.org/10.1088/0957-0233/13/3/312

[6] T. G. Leighton, D. G. Ramble, A.D. Phelps, C.L. Morfey, and P.P. Harris, "Acoustic detection of gas bubbles in a pipe", Acta Acustica united with Acustica, volume 84, issue 5, pages 801-814, 1998.

[7] X. Wu, G. L. Chahine, "Development of an acoustic instrument for bubble size distribution measurement", Journal of Hydrodynamics, Ser. B, Volume 22, Issue 5, Supplement 1, Pages 330-336, October, 2010. https://doi.org/10.1016/S1001-6058(09)60214-6 
[8] M. A. Ainslie, T. G. Leighton, "Review of scattering and extinction cross-sections, damping factors, and resonance frequencies of a spherical gas bubble", The Journal of the Acoustical Society of America, 2011, 130, pages 3184-3208. https://doi.org/10.1121/ 1.3628321

[9] A. Nuruzzaman, O. Boyraz, B. Jalali. "Time Stretched Short Time Fourier Transform". IEEE Transactions on Instrumentation and Measurement, Volume 55, Issue 2, 2006, pages 598-602. https://doi.org/10.1109/TIM.2006.864246

[10] W. B. Hussein, "Digital Processing Based Solutions for Life Science Engineering Recognition Problems", Ph.D. Thesis, Technische Universität München, Germany, 2013.

[11] G S Ranjitha, S M Pallavi. "Linear Predictive Coding". Proceedings of $8^{\text {th }}$ IRF international conference, 22/11/2015, Hyderabad, India, 2015, pages $46-49$.

[12] H. B. Demuth, M. H. Beale, O. D. Jesus. "Neural Network Design". $2^{\text {nd }}$ edition, Martin Hagan Press, 2014, pages 437-485.

\section{Authors}

W. B. Hussein and S. A. Essmat are with the Faculty of Informatics and Computer Science, The British University in Egypt, Cairo, Egypt (e-mails: walid.hussein | sarah.akram@bue.edu.eg).

Nestor B. Yoma with Department of Electrical Engineering, Universidad de Chile, Santiago, Chile.

This article is a revised version of a paper presented at the BUE International Conference on Sustainable Vital Technologies in Engineering and Informatics, held Nov 07, 2016 - Nov 09, 2016 , in Cairo, Egypt. Article submitted 21 December 2016. Published as resubmitted by the authors 23 February 2017. 